\title{
Trends in 10-Year Cardiovascular Disease Risk Among Adults in The United States From 1999-2016
}

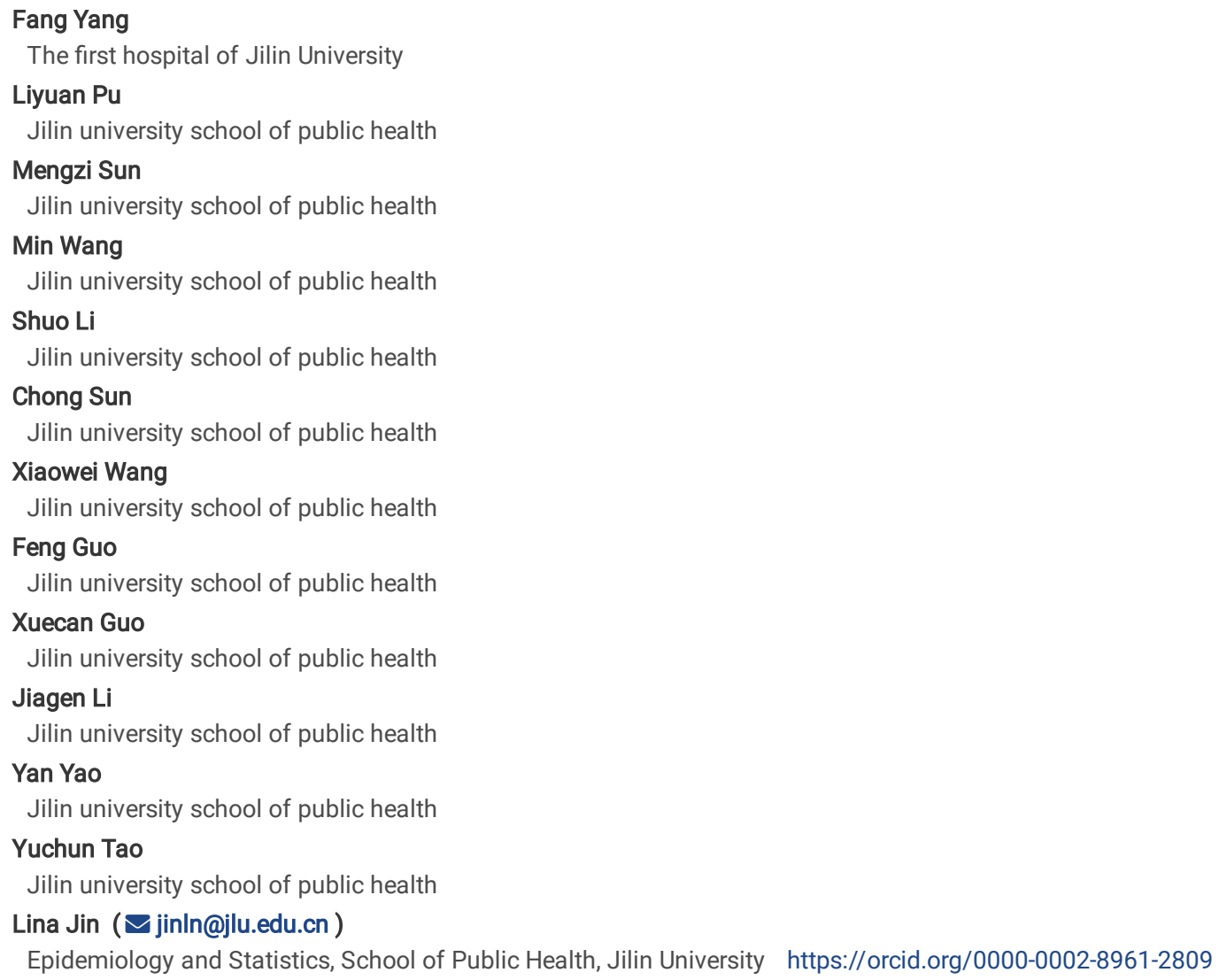

\section{Research article}

Keywords: Cardiovascular disease risk, Physical activity, Obesity

Posted Date: July 27th, 2020

DOI: https://doi.org/10.21203/rs.3.rs-42158/v1

License: (1) This work is licensed under a Creative Commons Attribution 4.0 International License. Read Full License 


\section{Abstract}

Objective: People have high 10-year cardiovascular disease (CVD) risk should take measures to prevent CVD. Thus, to know which subgroups of the population have high CVD risk is important. This study found out trends and risk factor of CVD risk in the US population.

Methods: The population aged 30 years and older who were not diagnosed with CVD came from the National Health and Nutrition Examination Surveys (NHANES) in this study, $n=35196$. Logistic regression models were used to analyze the trends of CVD risk and estimate association between risk factor and CVD risk.

Results: The estimated rate of CVD risk > 10\% changed from 1999 to 2016 (from $42.2 \%$ to $47.7 \%$ for males, from $24.7 \%$ to $23.9 \%$ for females). The estimated rate increased in males over time ( $P$ for trend $=0.001$ ), while it was stable in females. Males who did not participate in vigorous recreational activity, the estimated rate of CVD risk $>10 \%$ significantly increased ( $P$ for trend $<0.001$ ). The estimated rate of CVD risk $>10 \%, 20 \%$ and $30 \%$ increased with BMI level in males in the multivariable-adjusted model; the odds ratios and $95 \%$ confidence intervals (OR [95\% $\mathrm{Cl}]$ ) of obesity was 1.63 [1.41, 1.88 ] for $\mathrm{CVD}$ risk $>10 \%$, $1.69[1.45,1.48]$ for CVD risk $>20 \%$, and $1.56[1.30,1.87)]$ for CVD risk $>30 \%$.

Conclusions: The rate of CVD risk $>10 \%$ had an increasing trend in American males adults, who did not participate in vigorous recreational activity. In addition, lower annual household income, higher weight status and do not participate in vigorous recreational activity increased the CVD risk.

\section{Introduction}

It is widely accepted that cardiovascular disease (CVD) constitutes a main public health problem worldwide[1, 2]. Age-adjusted death rates attributed to overall CVD had a whole declined from 2000 to 2010 in the United States (US). However, mortality of CVD still accounted for one third of adult mortality[3]. CVD is the leading cause of morbidity and mortality in the elderly all over the world[4]. It is reported that the burden of CVD is a key determinant of the decline in the quality of life of people. In addition, the occurrence of CVD is usually silent or without warning, it is extremely important to prevent CVD for people who have high CVD risk[4, 5].

Nearly half of the US adults have at least one risk factor of CVD, and risk factor includes high blood pressure, high cholesterol and smoking and so on[6, 7]. If people with high CVD risk are found, early measures can be taken to prevent CVD for them[8]. Using risk prediction algorithms to predict high-risk populations and take targeted measures to avoid CVD is a key strategy for primary prevention of CVD [3,9]. The CVD risk assessment can identify people who have high CVD risk and provide counseling to individuals with treatable risk factors or unhealthy behaviors Besides, it shows low-risk individuals that they have done well[10].

The Framingham risk score (FRS) is a simplified and common tool for the assessment of 10-year CVD risk (CVD risk). It can assess conveniently general CVD risk by a sex-specific multivariable risk factor algorithm[11]. The FRS is recommended by other North American guidelines due to calculation of FRS is simple and fast[12]. Different FRS scores indicate different severity of CVD. Not only can it guide the prevention of CVD, but this score is also related to other diseases[13, 14]. A high score also indicates a high risk of other diseases. It is widely accepted that people with CVD risk $\geq 10 \%$ are generally the key population for health education, prevention and treatment of CVD, and CVD risk > 20\% are more likely to be have CVD[12].

People have high CVD risk should take measures to prevent CVD, it is important to know which subgroups of the population have high CVD risk. The estimates prevalence of CVD is described, but trends in CVD risk remain poorly described in the US population[15]. In addition, the trends of CVD risk under sociodemographic characteristic have not been systematically described in the population. Trends in CVD risk are explored according to sociodemographic and lifestyle characteristics in the US population, utilizing data from the National Health and Nutrition Examination Surveys (NHANES).

\section{Methods}

\section{Study Population}

The data of NHANES is representatives sample of the non-institutionalized civilian population of the US, due to used a complex, multistage probability sampling design. The investigation procedure was formal and approved by the National Center for Health Statistics ethics review committee[16,17]. This investigation used data from US adults aged 30 years and older during 9 cycles of NHANES from 1999 to 2016. If participants reported that they had never been diagnosed with congestive heart failure, coronary heart disease, angina/angina pectoris, heart attack, or stroke by a doctor or other health professional, we regarded them as normal populations. The covariates that make up the CVD risk are age, total cholesterol (TC), high density lipoprotein cholesterol (HDLC), systolic blood pressure (SBP), antihypertensive medication use, current smoking and diabetes status. The missing variables that constituted the CVD risk were assigned zero points[11]. All trends were examined by race/ethnicity, annual household income, educational attainment, vigorous recreational activity, moderate recreational activity and weight status[18].

\section{Assessment of CVD risk}

FRS provides score sheets that can be used for estimating CVD risk for males and females in normal populations, respectively. The corresponding score was assigned according to the value of age, TC $(\mathrm{mg} / \mathrm{dl}), \mathrm{HDL}-\mathrm{C}(\mathrm{mg} / \mathrm{dl})$, SBP, smoker and diabetic of the individual. The total score of all items corresponds to the CVD risk[11]. Self-reported treatment of hypertension and use of antihypertensive drugs were regarded as variable have been treated for hypertension.

Previous and present smoking is considered to be smoking. After a doctor's diagnosis, participant taking insulin or hypoglycemic drugs are considered to have diabetes. In addition, participant with combining fasting glucose $\geq 126(\mathrm{mg} / \mathrm{dl})$ or glycosylated hemoglobin $\geq 6.5$ (\%) are also considered to have 
diabetes[19]. According to different literatures, the criteria for CVD risk were divided into three levels for trend exploration: CVD risk > 10\%, CVD risk $>20 \%$ and CVD risk> 30\%[11, 20].

\section{Assessment of Sociodemographic and Lifestyle Characteristics}

Race/ethnicity was defined as non-Hispanic white, non-Hispanic black, Hispanic and other according to participant self-report. Annual household income fell into three categories $<\$ 25000, \$ 25000-\$ 74999$ and $\geq \$ 75000$. Educational attainment was divided into three categories (<High School, High School, $>$ High School). Moderate recreational activity and vigorous recreational activity were binary variable (yes or no). Weight status was measured during the physical examination at the mobile examination center, and body mass index (BMI) was calculated by weight $(\mathrm{kg})$ divided by height $(\mathrm{m})$ squared. Weight status was classified into normal $(18.5$ to $<25.0)$, overweight $(25.0$ to $<30.0)$, and obese $(\geq 30.0)$ group in adults.

\section{Statistical Analyses}

All analyses were adjusted weights, stratification and clustering of the complex sampling design. Logistic regression models were used to analyze the trends of CVD risk and estimate association between risk factor and CVD risk. All analyses were grouped by sex, due to the different scoring systems for males and females. The weighted rate, odds ratios (OR) and 95\% confidence intervals ( $95 \% \mathrm{Cl}$ ) of participants who are CVD risk $>10 \%$, CVD risk $>20 \%$ or CVD risk $>30 \%$ were calculated by race/ethnicity, annual household income, education attainment, vigorous recreational activity, moderate recreational activity and weight status, respectively.

The linear temporal trends were tested by multivariable logistic regression models. Survey cycles as continuous variable to calculate $P$ for trend by using survey-weighted logistic regression. Statistical tests were 2-sided and statistical significance was set at $P<0.05$. In the results presentation, a $P$ for trend $<$ 0.05 and $\beta>0$ an 'increase', a $P$ for trend $<0.05$ and $\beta<0$ represent a 'decrease', and $P$ for trend $\geq 0.05$ represent 'stable'. SPSS (Version 24.0 , IBM SPSS) was used for data analysis in this study.

\section{Results}

A total of 35196 individuals (mean age, 50.52 years [SE, 0.15 years]; 18525 [53.07\%] females) were analyzed in this study. Unweighted sample sizes overall and the trends of each composition of CVD risk for each sex group were presented in the Supplement (sTable1-2 and sFigure1). The estimated rate of CVD risk > 10\% changed from 1999 to 2016 (from 42.2-47.7\% for males, from $24.7-23.9 \%$ for females). The median of CVD risk of males was $12.66 \%$, showing $47.71 \%$ for CVD risk $>10 \%, 25.79 \%$ for CVD risk > 20\% and $12.51 \%$ for CVD risk > 30\% in the 2015-2016 cycle (Table 1). Besides, the median of CVD risk was $7.31 \%$, showing $23.85 \%$ for CVD risk $>10 \%, 9.33 \%$ for CVD risk $>20 \%$ and $2.13 \%$ for CVD risk $>30 \%$ of females. The $1999-2016$ estimated rate of CVD risk > $10 \%$ in males increased over time ( $P$ for trend $=0.001)$, while the estimated rate for CVD risk $>20 \%$ and $>30 \%$ in males were stable over time $(P$ for trend $=$ 0.071 and $P$ for trend $=0.171)$. The 1999-2016 estimated rate of CVD risk $>20 \%$ and $>30 \%$ in females decreased over time $(P$ for trend $=0.004$ and $P$ for trend $=0.001)$, while the estimated rate for CVD risk $>10 \%$ was stable over time $(P$ for trend $=0.364)($ Fig. 1$)$. 
Table 1

Crude weighted trends in 10-year CVD risk in the US population, NHANES 1999-2016 ${ }^{\text {a }}$

\begin{tabular}{|c|c|c|c|c|c|c|c|c|c|}
\hline $\begin{array}{l}\text { The 10- } \\
\text { year } \\
\text { CVD risk }\end{array}$ & $\begin{array}{l}1999-2000 \\
(\mathrm{~N}=3453)\end{array}$ & $\begin{array}{l}2001-2002 \\
(\mathrm{~N}=3762)\end{array}$ & $\begin{array}{l}2003-2004 \\
(\mathrm{~N}=3447)\end{array}$ & $\begin{array}{l}2005-2006 \\
(\mathrm{~N}=3361)\end{array}$ & $\begin{array}{l}2007-2008 \\
(N=4326)\end{array}$ & $\begin{array}{l}2009-2010 \\
(N=4518)\end{array}$ & $\begin{array}{l}2011-2012 \\
(\mathrm{~N}=3992)\end{array}$ & $\begin{array}{l}2013-2014 \\
(\mathrm{~N}=4222)\end{array}$ & $\begin{array}{l}2015-2016 \\
(N=4115)\end{array}$ \\
\hline \multicolumn{10}{|l|}{ Males } \\
\hline \multirow[t]{2}{*}{$\%$} & 1598(11.59) & 1764(11.64) & 1633(11.50) & 1644(11.29) & $2075(12.00)$ & 2129(11.93) & 1934(12.53) & 1973(12.16) & 1921(12.66) \\
\hline & {$[10.89,12.28]$} & {$[11.01,12.28]$} & {$[11.07,11.93]$} & {$[10.41,12.17]$} & {$[11.36,12.64]$} & {$[11.44,12.42]$} & {$[11.53,13.52]$} & {$[11.61,12.7]$} & {$[11.81,13.51]$} \\
\hline \multirow[t]{2}{*}{$>10 \%$} & $901(42.16)$ & $970(43.03)$ & $902(42.45)$ & $827(40.82)$ & 1139(45.13) & 1132(44.53) & 1038(48.22) & $1003(46.49)$ & 1039(47.71) \\
\hline & {$[39.08,45.31]$} & {$[39.36,46.79]$} & {$[39.56,45.39]$} & {$[35.55,46.31]$} & {$[40.70,49.64]$} & [42.04,47.04] & {$[43.51,52.97]$} & {$[43.11,49.89]$} & {$[43.89,51.55]$} \\
\hline \multirow[t]{2}{*}{$>20 \%$} & $597(23.54)$ & $587(21.20)$ & $588(23.27)$ & $510(21.37)$ & $689(22.66)$ & $672(22.40)$ & $612(24.53)$ & $580(23.57)$ & 634(25.79) \\
\hline & {$[21.4,25.82]$} & {$[18.61,24.03]$} & {$[21.74,24.87]$} & {$[18.52,24.54]$} & {$[20.38,25.12]$} & {$[20.45,24.49]$} & {$[21.29,28.10]$} & {$[21.30,26.01]$} & {$[22.05,29.93]$} \\
\hline \multirow[t]{2}{*}{$>30 \%$} & $314(10.96)$ & $307(9.84)$ & $318(10.73)$ & $242(9.01)$ & $359(10.63)$ & $331(9.77)$ & $304(10.91)$ & 283(10.68) & $327(12.51)$ \\
\hline & {$[9.68,12.38]$} & {$[8.37,11.52]$} & {$[8.99,12.75]$} & {$[7.59,10.66]$} & {$[9.29,12.15]$} & {$[8.54,11.15]$} & {$[8.57,13.79]$} & {$[9.24,12.32]$} & {$[9.97,15.6]$} \\
\hline \multicolumn{10}{|l|}{ Females } \\
\hline \multirow[t]{2}{*}{$\%$} & $1855(7.22)$ & 1998(6.94) & $1814(6.37)$ & $1717(6.62)$ & $2251(6.88)$ & 2389(6.92) & 2058(6.73) & $2249(6.96)$ & 2194(7.31) \\
\hline & {$[6.42,8.01]$} & {$[6.45,7.44]$} & {$[6.06,6.67]$} & {$[6.02,7.22]$} & {$[6.53,7.23]$} & {$[6.57,7.27]$} & {$[6.26,7.19]$} & {$[6.61,7.31]$} & {$[6.76,7.86]$} \\
\hline \multirow[t]{2}{*}{$>10 \%$} & $582(24.74)$ & $595(23.21)$ & $548(22.25)$ & $435(20.88)$ & 627(20.77) & $625(20.87)$ & 498(20.47) & $552(21.52)$ & 607(23.85) \\
\hline & {$[20.99,28.91]$} & {$[21.03,25.54]$} & {$[20.37,24.24]$} & [18.12,23.93] & {$[18.94,22.73]$} & [18.82,23.08] & {$[17.88,23.32]$} & {$[19.45,23.75]$} & [21.06,26.89] \\
\hline \multirow[t]{2}{*}{$>20 \%$} & $303(12.05)$ & $269(10.15)$ & $227(8.23)$ & $194(9.27)$ & $274(8.32)$ & $276(8.42)$ & $174(6.21)$ & $217(8.08)$ & $239(9.33)$ \\
\hline & {$[10.08,14.33]$} & {$[8.61,11.93]$} & {$[7.03,9.62]$} & {$[8.1,10.59]$} & {$[7.51,9.21]$} & {$[7.11,9.93]$} & {$[4.54,8.45]$} & {$[6.93,9.38]$} & {$[7.53,11.5]$} \\
\hline \multirow[t]{2}{*}{$>30 \%$} & $126(5.02)$ & $97(3.38)$ & $81(2.35)$ & $61(2.41)$ & 114(3.26) & $81(2.51)$ & $56(1.81)$ & $84(3.24)$ & $69(2.13)$ \\
\hline & {$[3.8 .00,6.59]$} & {$[2.55,4.48]$} & {$[1.65,3.34]$} & {$[1.82,3.19]$} & {$[2.52,4.22]$} & {$[1.94,3.24]$} & {$[1.35,2.43]$} & {$[2.61,4.03]$} & {$[1.58,2.88]$} \\
\hline
\end{tabular}

The 1999-2016 estimated rate of CVD risk $>10 \%$ increased over time in males who did not carry on vigorous recreational activity $(P$ for trend $<0.001)($ Table 2 and Fig. 2). Simultaneously, rate of CVD risk $>10 \%$ had significantly increase in males who did not carry on moderate recreational activity $(P$ for trend $<0.001)$. The estimated rate of CVD risk $>10 \%$ was stable in the obese males ( $P$ for trend $=0.449$ ). Males, whose educational attainment is high school, the estimated rate of CVD risk $>10 \%$ increased over time ( $P$ for trend $=0.007$ ). The estimated prevalence of CVD risk $>10 \%$ increased over time in males whose income are between $25,000 \$$ and $75,000 \$$ ( $P$ for trend $=0.001$ ). The estimates rate of CVD risk $>10 \%$ and $P$ for trend values for females was shown in sTable 3 of the Supplement. The estimates rate of CVD risk $>20 \%,>30 \%$ and $P$ for trend values between males and females was shown in sTable $4-7$ of the Supplement. 
Table 2

Crude weighted trends in 10-year CVD risk $>10 \%$, weight $\%(95 \% \mathrm{Cl})$ in the males US population, NHANES 1999-20.

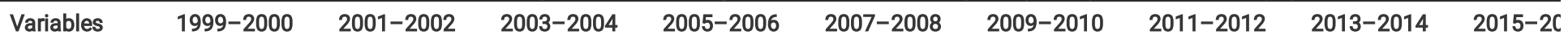

Race

\begin{tabular}{|c|c|c|c|c|c|c|c|c|c|}
\hline \multirow{2}{*}{$\begin{array}{l}\text { Non,Hispanic } \\
\text { White }\end{array}$} & $444(45.38)$ & $531(43.67)$ & 498(44.33) & $456(43.04)$ & $568(47.54)$ & $549(47.20)$ & $386(50.81)$ & $408(49.40)$ & $361(49.5$ \\
\hline & {$[40.98,49.85]$} & {$[39.34,48.11]$} & {$[40.04,48.7]$} & {$[36.65,49.68]$} & {$[41.78,53.37]$} & {$[43.85,50.56]$} & {$[45.4,56.19]$} & {$[44.34,54.47]$} & {$[44.12,54$} \\
\hline \multirow{2}{*}{$\begin{array}{l}\text { Non,Hispanic } \\
\text { black }\end{array}$} & 154(37.13) & 198(45.54) & $165(42.22)$ & 194(43.37) & $228(42.97)$ & $229(46.60)$ & 295(49.58) & 235(47.88) & $227(50.1$ \\
\hline & {$[31.8,42.80]$} & {$[39.87,51.33]$} & {$[36.57,48.09]$} & {$[37.32,49.62]$} & {$[36.49,49.7]$} & {$[39.97,53.35]$} & {$[42.44,56.73]$} & {$[41.63,54.20]$} & {$[44.58,55$} \\
\hline \multirow[t]{2}{*}{ Hispanic } & 281(32.19) & 209(34.71) & 214(36.82) & 152(24.50) & 294(34.85) & $303(34.24)$ & 208(36.44) & 232(35.83) & $308(39.2$ \\
\hline & {$[25.04,40.28]$} & {$[29.63,40.17]$} & {$[32.59,41.26]$} & {$[20.89,28.51]$} & {$[28.7,41.54]$} & {$[29.03,39.86]$} & {$[30.12,43.27]$} & {$[30.09,42.00]$} & {$[32.72,46$} \\
\hline \multirow[t]{2}{*}{ other ${ }^{d}$} & 22(31.49) & $32(49.92)$ & 25(28.09) & 25(39.76) & 49(43.17) & 51(33.39) & 149(44.34) & 128(39.83) & $143(46.3$ \\
\hline & {$[17.54,49.83]$} & {$[38.41,61.43]$} & {$[16.61,43.37]$} & [22.01,60.69] & {$[34.08,52.74]$} & {$[24.06,44.23]$} & {$[36.79,52.15]$} & [32.48,47.67] & {$[35.3,57$.} \\
\hline
\end{tabular}

Vigorous recreational activity

\begin{tabular}{|c|c|c|c|c|c|c|c|c|c|}
\hline \multirow[t]{2}{*}{ No } & $663(47.13)$ & 702(51.36) & $712(49.62)$ & $609(47.35)$ & 1005(53.69) & $1004(52.72)$ & $904(56.01)$ & 872(53.77) & $867(55.5$ \\
\hline & {$[42.71,51.6]$} & {$[47.35,55.34]$} & {$[45.18,54.07]$} & {$[42.42,52.33]$} & {$[48.42,58.89]$} & {$[49.24,56.18]$} & {$[51.66,60.28]$} & {$[50.12,57.38]$} & {$[51.35,5$} \\
\hline \multirow[t]{2}{*}{ Yes } & $184(32.21)$ & $225(30.15)$ & $134(25.22)$ & $172(27.25)$ & 134(22.05) & $128(21.41)$ & 133(27.81) & 131(25.02) & 172(30.1 \\
\hline & {$[25.43,39.83]$} & {$[24.24,36.81]$} & {$[21.28,29.61]$} & {$[20.43,35.35]$} & {$[17.56,27.31]$} & {$[17.34,26.13]$} & {$[21.31,35.4]$} & {$[21.4,29.01]$} & [24.76 \\
\hline
\end{tabular}

Moderate recreational activity

\begin{tabular}{|c|c|c|c|c|c|c|c|c|c|}
\hline \multirow[t]{2}{*}{ No } & $531(39.63)$ & $549(47.91)$ & $446(45.58)$ & $396(44.12)$ & 775(47.95) & 722(49.19) & $663(54.49)$ & 611(47.89) & $646(52.2$ \\
\hline & {$[35.92,43.46]$} & {$[43.03,52.83]$} & {$[41.8,49.41]$} & {$[38.71,49.68]$} & {$[42.68,53.27]$} & {$[44.06,54.33]$} & {$[48.51,60.34]$} & {$[43.69,52.12]$} & {$[48.14,56$} \\
\hline \multirow[t]{2}{*}{ Yes } & $324(44.60)$ & 392(38.23) & $424(38.98)$ & 404(37.75) & $364(41.18)$ & 410(38.99) & $374(40.86)$ & $392(44.75)$ & $393(42.9$ \\
\hline & {$[39.47,49.85]$} & {$[32.98,43.77]$} & {$[35.28,42.81]$} & {$[31.23,44.74]$} & {$[36.18,46.36]$} & {$[33.73,44.52]$} & {$[35.14,46.84]$} & {$[41.28,48.27]$} & {$[38.69,47$} \\
\hline
\end{tabular}

Weight status ${ }^{\mathrm{e}}$

\begin{tabular}{|c|c|c|c|c|c|c|c|c|c|}
\hline \multirow[t]{2}{*}{ Normal } & 211(35.09) & 199(30.11) & 197(34.31) & $190(36.52)$ & $228(36.53)$ & 233(37.48) & $247(45.74)$ & 229(41.91) & $223(41.3$ \\
\hline & {$[30.82,39.62]$} & {$[25.93,34.66]$} & [30.16,38.72] & {$[29.73,43.89]$} & {$[31.04,42.39]$} & [31.46,43.92] & {$[37.53,54.19]$} & [34.45,49.77] & {$[34.36,4 \varepsilon$} \\
\hline \multirow[t]{2}{*}{ Overweight } & $363(41.14)$ & $387(43.69)$ & $374(44.86)$ & $304(36.82)$ & $444(44.85)$ & $439(44.06)$ & $377(44.14)$ & $383(42.44)$ & $389(47.7$ \\
\hline & {$[36.05,46.42]$} & {$[39.22,48.26]$} & {$[39.73,50.11]$} & {$[30.97,43.09]$} & [38.72,51.15] & {$[39.92,48.28]$} & {$[38.96,49.44]$} & {$[38.73,46.24]$} & {$[40.31,55$} \\
\hline \multirow[t]{2}{*}{ Obesity } & $235(52.13)$ & $261(52.36)$ & $255(46.57)$ & 274(48.09) & $396(50.17)$ & $396(48.60)$ & $342(54.60)$ & $345(52.53)$ & $368(50.5$ \\
\hline & {$[46.93,57.28]$} & {$[48.52,56.17]$} & {$[41.95,51.26]$} & {$[41.8,54.44]$} & {$[42.61,57.72]$} & {$[43.4,53.83]$} & {$[47.2,61.81]$} & {$[47.84,57.18]$} & {$[46.00,55$} \\
\hline \multicolumn{10}{|c|}{ Educational level } \\
\hline \multirow[t]{2}{*}{$<$ High school } & $589(46.22)$ & $562(48.40)$ & $537(46.41)$ & $486(44.74)$ & $676(50.90)$ & 648(51.94) & $538(49.77)$ & $513(48.80)$ & $543(48.3$ \\
\hline & {$[43.15,49.32]$} & {$[45.6,51.22]$} & {$[43.03,49.83]$} & {$[37.57,52.14]$} & {$[43.98,57.78]$} & {$[48.41,55.45]$} & {$[44.02,55.54]$} & {$[45.46,52.16]$} & {$[43.46,53$} \\
\hline \multirow[t]{2}{*}{ High school } & 159(42.89) & 189(39.59) & 208(43.64) & 195(42.35) & $253(45.36)$ & 264(43.42) & 256(53.54) & 253(49.81) & $267(50.7$ \\
\hline & {$[35.71,50.39]$} & {$[33.92,45.56]$} & {$[38.28,49.16]$} & {$[36.31,48.63]$} & {$[39.28,51.57]$} & {$[37.00,50.06]$} & {$[43.31,63.48]$} & {$[44.24,55.39]$} & {$[43.41,5 \varepsilon$} \\
\hline \multirow{2}{*}{$\begin{array}{l}\text { > High } \\
\text { school }\end{array}$} & $148(34.67)$ & 215(38.79) & 155(34.62) & 142(33.19) & $210(35.83)$ & 219(35.79) & $241(42.26)$ & $237(41.36)$ & $228(44.4$ \\
\hline & {$[29.07,40.74]$} & {$[32.89,45.04]$} & {$[28.44,41.37]$} & {$[24.15,43.68]$} & {$[30.76,41.23]$} & {$[30.93,40.97]$} & {$[36.71,48.01]$} & {$[36.3,46.60]$} & {$[37.98,51$} \\
\hline
\end{tabular}

${ }^{\text {a }}$ Weighted estimates and $95 \% \mathrm{Cls}$ were estimated for each survey cycle. All estimates were weighted to be nationally representative.

b The estimate $\beta, 95 \% \mathrm{Cl}$, and $\mathrm{P}$ for trend were calculated using logistic regression model that included the National Health and Nutrition Examination Survey ( variable. The estimate $\beta$ can be interpreted as the average percentage point change in prevalence every 2 years.

${ }^{\mathrm{c}} \mathrm{A}$ decrease corresponds to difference below zero.

d "Other" includes race/ethnicity other than non-Hispanic white, non-Hispanic black, or Hispanic, including multiracial.

e Weight status was defined by body mass index, calculated as weight in kilograms divided by height in meters squared. The US Centers for Disease Control : for males and females. Weight status was classified into normal weight $(18.5-<25.0)$, overweight $(25.0-<30.0)$, and obese $(\geq 30.0)$ were used for adults 30 yei 


\begin{tabular}{|c|c|c|c|c|c|c|c|c|c|}
\hline Variables & 1999-2000 & $2001-2002$ & $2003-2004$ & $2005-2006$ & $2007-2008$ & $2009-2010$ & 2011-2012 & $2013-2014$ & $2015-2 C$ \\
\hline \multicolumn{10}{|l|}{ Income } \\
\hline \multirow[t]{2}{*}{$<25000$} & $310(48.27)$ & $302(58.68)$ & $313(55.02)$ & $256(56.44)$ & $341(54.81)$ & $302(54.14)$ & $333(56.93)$ & $279(55.30)$ & $287(61.4$ \\
\hline & {$[42.89,53.7]$} & {$[51.52,65.49]$} & {$[46.93,62.85]$} & {$[46.88,65.55]$} & {$[48.78,60.70]$} & {$[48.13,60.03]$} & {$[52.03,61.70]$} & {$[49.04,61.39]$} & {$[54.9,67$.} \\
\hline \multirow{2}{*}{$\begin{array}{l}25000 \sim< \\
75000\end{array}$} & $306(42.54)$ & $397(41.50)$ & 403(46.08) & $363(43.56)$ & $469(48.39)$ & $488(48.41)$ & $364(51.55)$ & $389(51.58)$ & $394(49.3$ \\
\hline & {$[36.88,48.41]$} & {$[37.59,45.52]$} & {$[42.53,49.67]$} & {$[37.49,49.83]$} & {$[43.24,53.59]$} & {$[44.05,52.79]$} & {$[45.68,57.36]$} & {$[46,57.12]$} & {$[42.6,56$. } \\
\hline \multirow[t]{2}{*}{$\geq 75000$} & - & - & - & - & $236(36.45)$ & 233(37.9) & $236(42.72)$ & $246(39.48)$ & $236(41.6$ \\
\hline & & & & & {$[30.02,43.41]$} & {$[34.56,41.37]$} & {$[36.43,49.25]$} & {$[36.07,43]$} & {$[35.61,47$} \\
\hline
\end{tabular}

a Weighted estimates and $95 \% \mathrm{Cls}$ were estimated for each survey cycle. All estimates were weighted to be nationally representative.

b The estimate $\beta, 95 \% \mathrm{Cl}$, and $\mathrm{P}$ for trend were calculated using logistic regression model that included the National Health and Nutrition Examination Survey ( variable. The estimate $\beta$ can be interpreted as the average percentage point change in prevalence every 2 years.

${ }^{\mathrm{c}} \mathrm{A}$ decrease corresponds to difference below zero.

d "Other" includes race/ethnicity other than non-Hispanic white, non-Hispanic black, or Hispanic, including multiracial.

e Weight status was defined by body mass index, calculated as weight in kilograms divided by height in meters squared. The US Centers for Disease Control : for males and females. Weight status was classified into normal weight $(18.5-<25.0)$, overweight $(25.0-<30.0)$, and obese $(\geq 30.0)$ were used for adults 30 yei

The estimated rate of CVD risk > 10\%, 20\% and 30\% decreased with increasing education level in females; the OR [95\% Cl] of > high school was 0.49 [0.42, 0.58 ] for CVD risk $>10 \%, 0.49[0.39,0.61]$ for CVD risk $>20 \%$, and $0.44[0.29,0.66]$ for CVD risk $>30 \%$, while the relationship did not exist between education level and CVD risk in males (Table 3). Additionally, the estimated rate of CVD risk > 10\%, 20\% and 30\% decreased with increasing annual household incomes in males; the OR $[95 \% \mathrm{Cl}]$ of $\geq 75000$ was $0.51[0.44,0.59]$ for CVD risk $>10 \%, 0.38[0.31,0.45]$ for CVD risk $>20 \%$, and $0.31[0.23,0.41]$ for CVD risk $>30 \%$. The relationship in males was same as females. The estimated rate of CVD risk $>10 \%, 20 \%$ and $30 \%$ increased with increasing $\mathrm{BMI}$ level in males; the OR [95\% $\mathrm{Cl}$ ] of obesity was $1.63[1.41,1.88]$ for CVD risk $>10 \%, 1.69[1.45,1.48]$ for CVD risk $>20 \%$, and $1.5[1.30,1.87]$ for CVD risk $>30 \%$. The relationship in males was same as females. Compared with their physically inactive counterparts, the OR [ $95 \% \mathrm{Cl}]$ of CVD risk $>10 \%, 20 \%$ and $30 \%$ for vigorous recreational activity people was $0.37[0.33,0.42], 0.35[0.30,0.41]$ and $0.29[0.23,0.36]$ in males. 
Table 3

Weighted logistic regression models of 10-year CVD risk $>10 \%$, adjusted for sociodemographic and lifestyle characteristics, NHANES $1999-2016$ a

Variables

Odds Ratio[95\% Cl] $]^{\text {b }}$

The 10 -year CVD risk $>10 \%$, Weight $\%(95 \% \mathrm{Cl}) \quad$ The 10 -year CVD risk $>20 \%$, Weight $\%$

$(95 \% \mathrm{Cl})$

The 10-year CVD risk $>30 \%$, Weight\% $(95 \% \mathrm{Cl})$

$\begin{array}{llllll}\text { Males } & \text { Females } & \text { Males } & \text { Females } & \text { Males } & \text { Females }\end{array}$

Race

\begin{tabular}{lllllll}
$\begin{array}{l}\text { Non,Hispanic } \\
\text { White }\end{array}$ & 1 [reference] & 1 [reference] & 1 [reference] & 1 [reference] & $1[$ reference] & $1[$ reference] \\
$\begin{array}{l}\text { Non,Hispanic } \\
\text { black }\end{array}$ & $0.825[0.729,0.933]$ & $0.674[0.601,0.755]$ & $0.838[0.741,0.948]$ & $0.689[0.579,0.821]$ & $0.852[0.746,0.974]$ & $0.729[0.569,0.936]$ \\
\hline Hispanic & $0.456[0.400,0.520]$ & $0.400[0.344,0.465]$ & $0.467[0.413,0.528]$ & $0.472[0.393,0.567]$ & $0.516[0.442,0.601]$ & $0.457[0.349,0.597]$ \\
\hline other & $0.821[0.664,1.015]$ & $0.717[0.567,0.908]$ & $0.810[0.657,0.997]$ & $0.727[0.551,0.961]$ & $0.766[0.587,0.999]$ & $0.835[0.536,1.298]$
\end{tabular}

Educational attainment

\begin{tabular}{lllllll} 
<High school & 1 [reference] & 1 [reference] & 1 [reference] & $1[$ reference] & 1 [reference] & $1[$ reference] \\
High school & $0.980[0.861,1.116]$ & $0.660[0.590,0.738]$ & $0.933[0.824,1.056]$ & $0.661[0.562,0.778]$ & $0.939[0.803,1.097]$ & $0.729[0.559,0.950]$ \\
\hline > High school & $0.933[0.804,1.081]$ & $0.493[0.418,0.581]$ & $0.947[0.809,1.109]$ & $0.488[0.392,0.606]$ & $0.861[0.698,1.062]$ & $0.438[0.291,0.659]$ \\
\hline Pfor trend $^{c}$ & 0.587 & $<0.001$ & 0.407 & $<0.001$ & 0.153 & $<0.001$
\end{tabular}

Income

\begin{tabular}{lllllll}
$<25000$ & $1[$ reference] & 1 [reference] & 1 [reference] & 1 [reference] & $1[$ reference] & $1[$ reference] \\
$\begin{array}{llllll}25000 \sim< \\
75000\end{array}$ & $0.701[0.622,0.790]$ & $0.590[0.524,0.664]$ & $0.651[0.579,0.731]$ & $0.506[0.430,0.596]$ & $0.613[0.539,0.696]$ & $0.461[0.360,0.590]$ \\
$\geq 75000$ & $0.509[0.437,0.593]$ & $0.307[0.255,0.368]$ & $0.375[0.312,0.449]$ & $0.266[0.204,0.348]$ & $0.306[0.231,0.406]$ & $0.186[0.111,0.312]$ \\
\hline$P$ for trend $^{c}$ & $<0.001$ & $<0.001$ & $<0.001$ & $<0.001$ & $<0.001$ & $<0.001$
\end{tabular}

Weight status ${ }^{\mathrm{d}}$

\begin{tabular}{lllllll} 
Normal & 1 [reference] & 1 [reference] & 1 [reference] & 1 [reference] & 1 [reference] & 1 [reference] \\
\hline Overweight & $1.354[1.177,1.556]$ & $1.400[1.237,1.584]$ & $1.379[1.203,1.581]$ & $1.393[1.174,1.651]$ & $1.400[1.183,1.657]$ & $1.412[1.054,1.891]$ \\
\hline obesity & $1.625[1.404,1.881]$ & $1.467[1.285,1.675]$ & $1.687[1.446,1.967]$ & $1.246[1.050,1.477]$ & $1.555[1.296,1.866]$ & $1.105[0.835,1.463]$ \\
\hline Pfor trend & $<0.001$ & $<0.001$ & $<0.001$ & 0.029 & $<0.001$ & 0.975
\end{tabular}

Vigorous recreational activity

$\begin{array}{lllllll}\text { No } & 1 \text { [reference] } & 1 \text { [reference] } & 1 \text { [reference] } & 1 \text { [reference] } & 1[\text { reference] } & 1[\text { reference] } \\ \text { Yes } & 0.368[0.327,0.415] & 0.392[0.331,0.464] & 0.349[0.298,0.409] & 0.371[0.281,0.490] & 0.287[0.229,0.358] & 0.235[0.136,0.406]\end{array}$

CYCLE

\begin{tabular}{lllllll|} 
1999-2000 & 1 [reference] & 1 [reference] & 1 [reference] & 1 [reference] & 1 [reference] & 1 [reference] \\
\hline $2001-2002$ & $1.047[0.809,1.354]$ & $0.971[0.792,1.190]$ & $0.869[0.689,1.096]$ & $0.887[0.676,1.163]$ & $0.855[0.649,1.126]$ & $0.752[0.467,1.210]$ \\
\hline $2003-2004$ & $1.062[0.824,1.370]$ & $0.880[0.703,1.102]$ & $1.015[0.826,1.248]$ & $0.655[0.507,0.845]$ & $0.884[0.654,1.194]$ & $0.434[0.262,0.718]$ \\
\hline $2005-2006$ & $1.007[0.733,1.383]$ & $0.975[0.764,1.245]$ & $0.961[0.756,1.222]$ & $0.872[0.668,1.138]$ & $0.849[0.618,1.166]$ & $0.554[0.360,0.852]$ \\
\hline $2007-2008$ & $1.080[0.830,1.406]$ & $0.851[0.702,1.030]$ & $0.947[0.768,1.169]$ & $0.702[0.550,0.895]$ & $0.969[0.762,1.232]$ & $0.662[0.437,1.002]$ \\
\hline
\end{tabular}

a Participant characteristics were presented by sex group: males and females. All estimates were weighted to be nationally representative.

${ }^{b}$ For categorical variables, the odds ratios (ORs) represent the change in odds expected in each category compared with the reference group.

${ }^{\mathrm{c}} \mathrm{P}$ for trend over annual household income was calculated using the value of each category as a continuous variable.

${ }^{\mathrm{d}}$ For weight status definitions, see the Table 2 footnotes.

${ }^{\mathrm{e}}$ Calculated using BMI as a continuous variable.

${ }^{f} \mathrm{P}$ for trend over survey cycle was calculated using the National Health and Nutrition Examination Survey (NHANES) 2-year survey cycle as a continuous variable. 


\begin{tabular}{|c|c|c|c|c|c|c|}
\hline \multirow[t]{3}{*}{ Variables } & \multicolumn{6}{|l|}{ Odds Ratio[95\% Cl] $]^{b}$} \\
\hline & \multicolumn{2}{|c|}{ The 10-year CVD risk > 10\%, Weight\%(95\%Cl) } & \multicolumn{2}{|c|}{$\begin{array}{l}\text { The } 10 \text {-year CVD risk }>20 \% \text {, Weight } \% \\
(95 \% \mathrm{Cl})\end{array}$} & \multicolumn{2}{|c|}{$\begin{array}{l}\text { The } 10 \text {-year CVD risk }>30 \% \text {, Weight } \% \\
(95 \% \mathrm{Cl})\end{array}$} \\
\hline & Males & Females & Males & Females & Males & Females \\
\hline $2009-2010$ & $1.092[0.858,1.389]$ & $0.873[0.707,1.078]$ & $0.952[0.769,1.179]$ & $0.695[0.505,0.956]$ & $0.832[0.653,1.060]$ & $0.475[0.294,0.765]$ \\
\hline $2011-2012$ & $1.322[0.991,1.765]$ & $0.893[0.718,1.111]$ & $1.091[0.849,1.400]$ & $0.541[0.356,0.820]$ & $0.962[0.705,1.312]$ & $0.400[0.253,0.630]$ \\
\hline $2013-2014$ & $1.182[0.898,1.554]$ & $1.004[0.817,1.235]$ & $1.037[0.829,1.298]$ & $0.798[0.594,1.074]$ & $0.921[0.710,1.196]$ & $0.798[0.536,1.187]$ \\
\hline $2015-2016$ & $1.322[1.011,1.729]$ & $1.251[0.997,1.569]$ & $1.255[0.985,1.599]$ & $0.978[0.735,1.301]$ & $1.219[0.902,1.649]$ & $0.541[0.348,0.840]$ \\
\hline$P$ for trend $^{f}$ & 0.026 & 0.050 & 0.010 & 0.408 & 0.119 & 0.046 \\
\hline \multicolumn{7}{|c|}{ a Participant characteristics were presented by sex group: males and females. All estimates were weighted to be nationally representative. } \\
\hline \multicolumn{7}{|c|}{ b For categorical variables, the odds ratios (ORs) represent the change in odds expected in each category compared with the reference group. } \\
\hline \multicolumn{7}{|c|}{${ }^{\mathrm{c}} \mathrm{P}$ for trend over annual household income was calculated using the value of each category as a continuous variable. } \\
\hline \multicolumn{7}{|c|}{ d For weight status definitions, see the Table 2 footnotes. } \\
\hline \multicolumn{7}{|c|}{ e Calculated using BMI as a continuous variable. } \\
\hline
\end{tabular}

\section{Discussion}

The 1999-2016 estimated rate of CVD risk $>10 \%$ increased over time in males, while the estimated rate for CVD risk $>20 \%$ and $>30 \%$ in males were stable over time. Besides, the estimated rate of CVD risk did not show an increase during 1999-2016 for females. The 1999-2016 estimated rate of CVD risk > 10\% increased over time in males who did not carry on vigorous recreational activity and moderate recreational activity. The rate of CVD risk was lower for the people who participated in vigorous recreational activity than others. Additionally, males and females from lower-income families or higher-weight status have a higher CVD risk.

Epidemiologic and clinical studies have demonstrated that CVD prevention is feasible, realistic, and within reach. The CVD risk $>10 \%$ of males in this study is increasing, and people with CVD risk > 10\% should take the measures to prevent CVD. Elevated blood lipid levels[21], hypertension[22], elevated blood glucose levels, overweight[23], unhealthy dietary habits, cigarette smoking and insufficient regular exercise were established primary causal factors of CVD[5]. Moreover, the incidence of CVD increased rapidly in low or moderate income countries[7]. It is consistent with our findings in this study. Participants with lower income level should take measures to prevent CVD.

The prevalence of obesity-related CVD is rapidly increasing, although CVD may be caused by multiple factors[24]. In fact, obesity is an independent risk factor for CVD. Overweight and obesity are associated with many cardiac complications, such as coronary heart disease. Obesity usually leads to up-regulation of pro-inflammatory adipokines and down-regulation of anti-inflammatory adipokines, thereby contributing to the pathogenesis of CVD[25]. Hence, obesity may affect the heart through its influence on known risk factors such as dyslipidemia and hypertension[26]. Watching TV for a long time may exacerbate the genetic susceptibility of obesity, while longer leisure activities may weaken these associations[27]. These findings suggested that participate who was overweight or obese reach normal level by controlling weight may be conducive to the prevention of CVD.

Studies have shown that exercise can improve metabolic and cardiovascular health independent of changes in body weight, including improved glucose homeostasis, endothelial function, blood pressure, and HDL levels[28-30]. Studies have shown that lack of physical activity is a clear independent risk factor of CVD[31]. Compared with low physical activity, moderate and high physical activity were protect factors of CVD associated with graded reduction in mortality[32]. Physical activity was generally associated with more favorable cardiovascular biomarker levels than inactivity[33]. Several recent studies have shown that sustained physical activity was associated with decreased markers of inflammation, improved metabolic health, decreased risk of heart failure and improved overall survival[34]. A physical activity, especially recreational has a protective effect against CVD in high-income countries. Increasing physical activity is a simple, widely applicable, low-cost global strategy that can reduce the deaths and CVD of middle-aged people[35]. Vigorous recreational activity may be an effective measure to prevent the occurrence of CVD and the harm it brings.

\section{Limitation}

This study has a little limitation. CVD risk score may not fully reflect the level of CVD. Self-reported smoker and diabetes were used to assess the risk of CVD, but they have been widely used in epidemiological studies. And measurement errors were unlikely to affect findings on the secular trends over time in this study.

\section{Conclusion}


There was an increasing trend of CVD risk $>10 \%$ in American males adults. The increasing trend of CVD risk > 10\% was mainly manifested in males who did not participate in vigorous recreational activity. In addition, lower annual household incomes, higher weight status and do not participate in vigorous recreational activity increased the risk of CVD risk $>10 \%$. Participants with the above characteristics should not be neglected in the prevention of CVD. At the same time, policy-makers should pay more attention to the new tendency.

\section{Abbreviations}

CVD: Cardiovascular disease; US: United States; FRS: Framingham risk score; CVD risk: 10-year CVD risk; TC: total cholesterol; HDL-C: high density lipoprotein cholesterol; SBP: systolic blood pressure; BMI: body mass index; NHANES: National Health and Nutrition Examination Survey; OR: Odds ratio; Cl: Confidence interval.

\section{Declarations}

\section{Ethics approval and consent to participate}

The National Center for Health Statistics ethnics review board approved study programs for NHANES. All the participants signed the informed consent.

\section{Consent for publication}

Not applicable.

\section{Competing interests}

The authors declare that they have no competing interests.

\section{Funding}

This work was supported by the Natural Science Foundation of Science and Technology Development of Jilin Province, China (No. 20180101129JC) and the National Natural Science Foundation of China (No.81973129). The funders had no role in the design neither of the study nor in the collection, analysis, and interpretation of data or in writing the manuscript.

\section{Authors' contributions}

FY and LP contributed to the design of the work. LJ and YT contributed to the conception and design of the work. FY, LP, MS, MW, SL, CS, XW, FG, XG, JL, YY YT and LJ contributed to the acquisition, analysis, or interpretation of data for the work. FY, LP, MW, SL and XW drafted the manuscript. LJ, YT, YY, MS, CS, FG, $X G$ and $J L$ critically revised the manuscript. All gave final approval and agree to be accountable for all aspects of work ensuring integrity and accuracy.

\section{Acknowledgements}

The authors thank the National Center for Health Statistics of the Centers for Disease Control and Prevention for sharing the data.

\section{Data Availability Statement}

Data for this study are publicly available in https://www.cdc.gov/nchs/nhanes/.

\section{References}

1. Rosamond W, Flegal K, Friday G, Furie K, Go A, Greenlund K, et al. Heart disease and stroke statistics-2007 update: a report from the American Heart Association Statistics Committee and Stroke Statistics Subcommittee. Circulation. 2007;115:e69-171.

2. Lopez AD, Mathers CD, Ezzati M, Jamison DT, Murray CJL. Global and regional burden of disease and risk factors, 2001: systematic analysis of population health data. Lancet (London, England). 2006;367:1747-57.

3. Bibbins-Domingo K, Grossman DC, Curry SJ, Davidson KW, Epling JWJ, Garcia FAR, et al. Statin use for the primary prevention of cardiovascular disease in adults: US Preventive Services Task Force Recommendation Statement. JAMA. 2016;316:1997-2007.

4. Lloyd-Jones DM, Leip EP, Larson MG, D'Agostino RB, Beiser A, Wilson PWF, et al. Prediction of lifetime risk for cardiovascular disease by risk factor burden at 50 years of age. Circulation. 2006;113:791-8.

5. Lloyd-Jones DM, Hong Y, Labarthe D, Mozaffarian D, Appel LJ, Van Horn L, et al. Defining and setting national goals for cardiovascular health promotion and disease reduction: the American Heart Association's strategic Impact Goal through 2020 and beyond. Circulation. 2010;121:586-613.

6. Montori VM, Brito JP, Ting HH. Patient-centered and practical application of new high cholesterol guidelines to prevent cardiovascular disease. JAMA - J Am Med Assoc. 2014;311:465-6.

7. Greenland P, Peterson ED, Gaziano JM. Progress against cardiovascular disease: putting the pieces together. JAMA. 2014;312:1979-80.

8. Park B, Lee Y-J. Borderline high serum calcium levels are associated with arterial stiffness and 10-year cardiovascular disease risk determined by Framingham risk score. J Clin Hypertens (Greenwich). 2019;21:668-73.

9. Cooney MT, Dudina A, D’Agostino R, Graham IM. Cardiovascular risk-estimation systems in primary prevention: do they differ? Do they make a difference? Can we see the future? Circulation. 2010;122:300-10. 
10. Cooney MT, Dudina AL, Graham IM. Value and limitations of existing scores for the assessment of cardiovascular risk: a review for clinicians. J Am Coll Cardiol. 2009;54:1209-27.

11. D'Agostino RBS, Vasan RS, Pencina MJ, Wolf PA, Cobain M, Massaro JM, et al. General cardiovascular risk profile for use in primary care: the Framingham Heart Study. Circulation. 2008;117:743-53.

12. Anderson TJ, Gregoire J, Pearson GJ, Barry AR, Couture P, Dawes M, et al. 2016 Canadian cardiovascular society guidelines for the management of dyslipidemia for the prevention of cardiovascular disease in the Adult. Can J Cardiol. 2016;32:1263-82.

13. Joosten H, van Eersel MEA, Gansevoort RT, Bilo HJG, Slaets JPJ, Izaks GJ. Cardiovascular risk profile and cognitive function in young, middle-aged, and elderly subjects. Stroke. 2013;44:1543-9.

14. Di Maira T, Rubin A, Puchades L, Aguilera V, Vinaixa C, Garcia M, et al. Framingham score, renal dysfunction, and cardiovascular risk in liver transplant patients. Liver Transplant Off Publ Am Assoc Study Liver Dis Int Liver Transplant Soc. 2015;21:812-22.

15. Shay CM, Ning H, Allen NB, Carnethon MR, Chiuve SE, Greenlund KJ, et al. Status of cardiovascular health in US adults: prevalence estimates from the national health and nutrition examination surveys (NHANES) 2003-2008. Circulation. 2012;125:45-56.

16. Johnson CL, Paulose-Ram R, Ogden CL, Carroll MD, Kruszon-Moran D, Dohrmann SM, et al. National health and nutrition examination survey: analytic guidelines, 1999-2010. Vital Health Stat 2. 2013;:1-24.

17. Curtin LR, Mohadjer LK, Dohrmann SM, Montaquila JM, Kruszan-Moran D, Mirel LB, et al. The national health and nutrition examination survey: Sample Design, 1999-2006. Vital Health Stat 2. 2012;:1-39.

18. Borhanuddin B, Mohd Nawi A, Shah SA, Abdullah N, Syed Zakaria SZ, Kamaruddin MA, et al. 10-year cardiovascular disease risk estimation based on lipid profile-based and BMI-based Framingham risk scores across multiple sociodemographic characteristics: The Malaysian Cohort Project.

ScientificWorld Journal. 2018;2018:2979206.

19. Gregg EW, Cadwell BL, Cheng YJ, Cowie CC, Williams DE, Geiss L, et al. Trends in the prevalence and ratio of diagnosed to undiagnosed diabetes according to obesity levels in the U.S. Diabetes Care. 2004;27:2806-12.

20. Ford ES, Giles WH, Mokdad AH. The distribution of 10-year risk for coronary heart disease among US adults: findings from the national health and nutrition examination survey III. J Am Coll Cardiol. 2004;43:1791-6.

21. Stone NJ, Robinson JG, Lichtenstein AH, Bairey Merz CN, Blum CB, Eckel RH, et al. 2013 ACC/AHA guideline on the treatment of blood cholesterol to reduce atherosclerotic cardiovascular risk in adults: a report of the American College of Cardiology/American Heart Association Task Force on Practice Guidelines. J Am Coll Cardiol. 2014;63 25 Pt B:2889-934.

22. James PA, Oparil S, Carter BL, Cushman WC, Dennison-Himmelfarb C, Handler J, et al. 2014 evidence-based guideline for the management of high blood pressure in adults: report from the panel members appointed to the Eighth Joint National Committee (JNC 8). JAMA. 2014;311:507-20.

23. Jensen MD, Ryan DH, Apovian CM, Ard JD, Comuzzie AG, Donato KA, et al. 2013 AHA/ACC/TOS guideline for the management of overweight and obesity in adults: a report of the American College of Cardiology/American Heart Association Task Force on Practice Guidelines and The Obesity Society. J Am Coll Cardiol. 2014;63 25 Pt B:2985-3023.

24. Kivimaki M, Kuosma E, Ferrie JE, Luukkonen R, Nyberg ST, Alfredsson L, et al. Overweight, obesity, and risk of cardiometabolic multimorbidity: pooled analysis of individual-level data for 120813 adults from 16 cohort studies from the USA and Europe. Lancet Public Heal. 2017;2:e277-85.

25. Fuster JJ, Ouchi N, Gokce N, Walsh K. Obesity-induced changes in adipose tissue microenvironment and their impact on cardiovascular disease. Circ Res. 2016;118:1786-807.

26. Poirier P, Giles TD, Bray GA, Hong Y, Stern JS, Pi-Sunyer FX, et al. Obesity and cardiovascular disease: pathophysiology, evaluation, and effect of weight loss: an update of the 1997 American Heart Association Scientific Statement on Obesity and Heart Disease from the Obesity Committee of the Council on Nutrition, Physical. Circulation. 2006;113:898-918.

27. Circulation editors' picks: obesity and cardiovascular disease. Circulation. 2013;128:e432-42.

28. Church TS, Blair SN, Cocreham S, Johannsen N, Johnson W, Kramer K, et al. Effects of aerobic and resistance training on hemoglobin A1c levels in patients with type 2 diabetes: a randomized controlled trial. JAMA. 2010;304:2253-62.

29. Sigal RJ, Kenny GP, Boule NG, Wells GA, Prud'homme D, Fortier M, et al. Effects of aerobic training, resistance training, or both on glycemic control in type 2 diabetes: a randomized trial. Ann Intern Med. 2007;147:357-69.

30. Swift DL, Earnest CP, Blair SN, Church TS. The effect of different doses of aerobic exercise training on endothelial function in postmenopausal women with elevated blood pressure: results from the DREW study. Br J Sports Med. 2012;46:753-8.

31. Cornelissen VA, Fagard RH. Effects of endurance training on blood pressure, blood pressure-regulating mechanisms, and cardiovascular risk factors. Hypertens (Dallas, Tex 1979). 2005;46:667-75.

32. Grontved A, Hu FB. Television viewing and risk of type 2 diabetes, cardiovascular disease, and all-cause mortality: a meta-analysis. JAMA. 2011;305:2448-55.

33. Mora S, Lee I-M, Buring JE, Ridker PM. Association of physical activity and body mass index with novel and traditional cardiovascular biomarkers in women. JAMA. 2006;295:1412-9.

34. Che L, Li D. The effects of exercise on cardiovascular biomarkers: New Insights, Recent Data, and Applications. Adv Exp Med Biol. 2017;999:43-53.

35. Lear SA, Hu W, Rangarajan S, Gasevic D, Leong D, lqbal R, et al. The effect of physical activity on mortality and cardiovascular disease in 130000 people from 17 high-income, middle-income, and low-income countries: the PURE study. Lancet (London, England). 2017;390:2643-54.

\section{Figures}

Page 10/12 


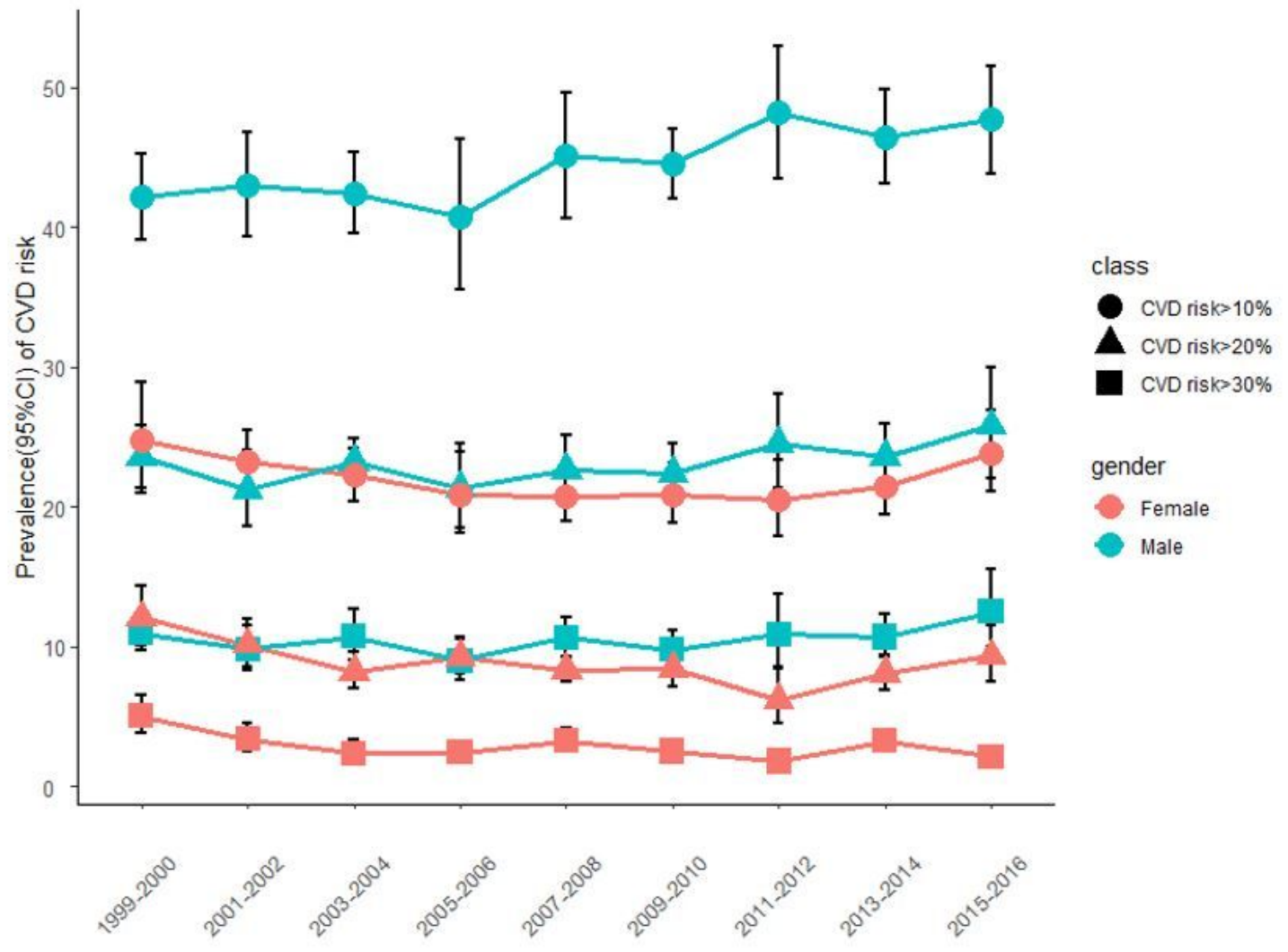

Figure 1

Crude Weighted Trends in 10-year CVD risk among the US Population, NHANES 1999-2016

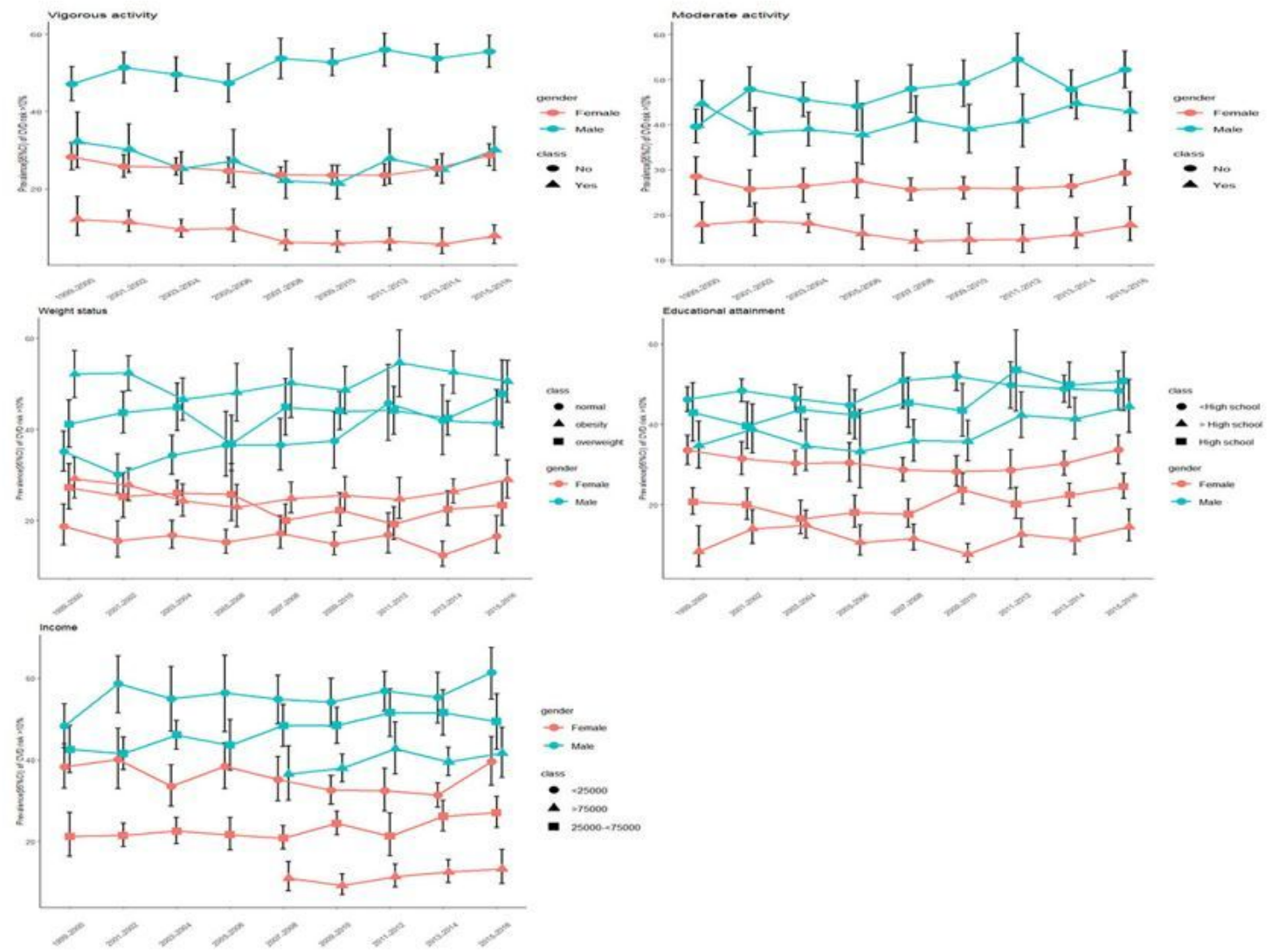

Figure 2 
Crude weighted trends in 10-year CVD risk >10\% according to vigorous recreational activity, moderate recreational activity, weight status, educational level and income, NHANES 1996-2016

\section{Supplementary Files}

This is a list of supplementary files associated with this preprint. Click to download.

- Supplementarymaterial.doc 